\title{
ASO Author Reflections: Applications of Artificial Intelligence in Oesophago-Gastric Malignancies-Present Work and Future Directions
}

\author{
Swathikan Chidambaram, MBBS, BSc, MRCS ${ }^{1}$, Viknesh Sounderajah, MBBS, BSc, MRCS ${ }^{1,2}$, \\ Nick Maynard, MBBS, BA, FRCS ${ }^{3}$, and Sheraz R. Markar, PhD (Imperial), PhD (Karolinska), MRCS, MSc, MA ${ }^{1,3,4}$ \\ ${ }^{1}$ Department of Surgery and Cancer, Imperial College London, London, UK; ${ }^{2}$ Institute of Global Health Innovation, \\ Imperial College London, London, UK; ${ }^{3}$ Department of Surgery, Churchill Hospital, Oxford University Hospitals NHS \\ Trust, Oxford, UK; ${ }^{4}$ Department of Molecular Medicine and Surgery, Karolinska Institutet, Stockholm, Sweden
}

\begin{abstract}
Our paper highlights the use of artificial intelligence (AI) in oesophageal and gastric malignancies with acceptable levels of accuracy for both diagnostic and surveillance purposes. Here, we comment on the past, present and future work necessary for incorporating AI into the clinical framework and practice.
\end{abstract}

\section{PAST}

Artificial intelligence (AI)-centred systems have shown promise in facilitating the diagnosis and management of many disease processes, including cancers. ${ }^{1}$ With an increase in the prevalence and mortality of oesophagogastric (OG) cancers, there is a particular need for enhanced methods of diagnosis in this space, particularly as a means of surveillance. As such, there is much fervour around the potential of AI techniques to be able to recognise features of malignancy on routine investigations that this cohort of patients undergo, including endoscopy, computed tomography (CT) scans, CT-positron emission tomography (PET) scans and magnetic resonance imaging (MRI) scans.

\section{(C) The Author(s) 2021}

First Received: 24 September 2021 Accepted: 24 September 2021;

Published Online: 18 November 2021

S. R. Markar, PhD (Imperial), PhD (Karolinska), MRCS, MSc, MA

e-mail: sheraz.markar@ouh.nhs.uk

\section{PRESENT}

Previous studies have shown varying degrees of success in developing AI systems to detect oesophageal and gastric cancers for both diagnostic and surveillance purposes. In our study, we have summarised their results and shown that there is considerable promise in this field, despite its relative nascency. In the quantitative synthesis, the pooled sensitivity and specificity for 1352 patients were $73.4 \%$ and $89.7 \%$, respectively. ${ }^{2}$ Moreover, the incorporation of radiomic techniques can further enhance the diagnostic accuracy of the process. Key limitations in the field currently centre around the absence of a randomised clinical trial to evaluate performance prospectively, and the reliance upon small retrospective studies which are susceptible to bias.

\section{FUTURE}

There is clearly an abundance of potential for using AI in the management of OG cancers. Currently, we are in an era that is shifting towards an organ-sparing approach, relying solely on primary oncological treatment and avoiding the morbidity of surgical intervention. This is only possible due to effective chemo-radiotherapy (CRT) regimes developed in the past decade. The ongoing SANO and NEEDS trials are evaluating the safety and feasibility of this strategy. ${ }^{3}$ However, this is only possible if recurrence or residual cancer can be accurately detected on surveillance. A well-designed AI system that is trained and validated to achieve high diagnostic accuracies will play an unprecedented role in achieving this. To do this, large 
datasets of patient data, including clinical details, endoscopy images and imaging, need to be collated encompassing a range of factors including demographic (age, sex, ethnicity, comorbidities), oncological (CRT regime), surgical (approach, technique, extent of lymphadenectomy) and tumour level (histology, pre-operative and pathological TNM stages, grade). The AI system will also require robust independent validation using external datasets. Furthermore, the translation of the AI model to clinical practice requires infrastructure to be implemented, while also taking into consideration its cost-effectiveness as well as acceptability by stakeholders, including clinicians and patients. All of the above strongly emphasise the need for well-designed randomised clinical trials conducted on a large scale through collaboration to fully realise the potentials of artificial intelligence systems.

DISCLOSURES We confirm that the work is original and has not been previously published in other mediums or under consideration for publication elsewhere, in its current form. Patients were not involved in the conception, design, analysis, drafting, interpretation or revision of this research. There have been no previous publications or presentations of this study, and this work did not require any funding. The authors have no conflicts of interest to declare.

OPEN ACCESS This article is licensed under a Creative Commons Attribution 4.0 International License, which permits use, sharing, adaptation, distribution and reproduction in any medium or format, as long as you give appropriate credit to the original author(s) and the source, provide a link to the Creative Commons licence, and indicate if changes were made. The images or other third party material in this article are included in the article's Creative Commons licence, unless indicated otherwise in a credit line to the material. If material is not included in the article's Creative Commons licence and your intended use is not permitted by statutory regulation or exceeds the permitted use, you will need to obtain permission directly from the copyright holder. To view a copy of this licence, visit http://creativecommons. org/licenses/by/4.0/.

\section{REFERENCES}

1. McKinney SM, Sieniek M, Godbole V, Godwin J, Antropova N, Ashrafian $\mathrm{H}$, et al. International evaluation of an AI system for breast cancer screening. Nature. 2020;577(7788):89-94.

2. Chidamabaram S, Sounderajah V, Maynard N, Markar SR. Diagnostic performance of artificial intelligence centred systems in the diagnosis and post-operative surveillance of upper gastrointestinal malignancies using ct imaging: a systematic review and meta-analysis of diagnostic accuracy. Ann Surg Oncol. 2021. https://doi.org/10.1245/s10434-021-10882-6.

3. Eyck BM, van der Wilk BJ, Noordman BJ, Wijnhoven BPL, Lagarde SM, Hartgrink HH, et al. Updated protocol of the SANO trial: a stepped-wedge cluster randomised trial comparing surgery with active surveillance after neoadjuvant chemoradiotherapy for oesophageal cancer. Trials. 2021;22(1):1-6.

Publisher's Note Springer Nature remains neutral with regard to jurisdictional claims in published maps and institutional affiliations. 